\title{
Can patch distribution affect diet selection by sheep?
}

\author{
B. Dumont, A. Pouillon, P. D'hour
}

Laboratoire adaptation des gerbivores aux milieux, Inra, 63122 Saint-Genès-Champanelle, France

As the availability of a preferred species decreases sheep switch to more abundant sward components, however there is no evidence that patch distribution has any additional effect on diet selection. We observed the choice, and subsequent impact on the sward, of three groups of four ewes in three 7.5 -cm-high cocksfoot plots $(80 \times 80 \mathrm{~m})$ containing 136 patches $(30 \times 30 \mathrm{~cm})$ of ryegrass known to be preferred patches. The patches were randomly distributed in the first plot (Rand) and were grouped together to create aggregates (four of 9 and four of 25 patches) in the other two plots (Ag.1 and $\mathrm{Ag} .2$ ). These plots differed in aggregate location and density. After $2 \mathrm{~h}$ of grazing, patches in plots Ag.1 and Ag.2 tended to be more exploited: their height was reduced by 20.5 and $19.5 \%$, respectively (versus $10.7 \%$ in plot Rand; $P<0.10$ ) and ewes had spent 12.8 and $13.1 \%$ of time on them (versus $10.6 \%$; NS). Behavioural observations showed that once the ewes had discovered an aggregate in plot Ag.1 or Ag. 2 they returned to it several times, whereas they seemed unable to learn the precise location of many isolated patches in plot Rand. Patch distribution can thus affect diet selection. Grazing behaviour differed across groups $(P<0.05)$ : time spent grazing on ryegrass was not affected by patch distribution in one group (Rand: $15.5 \%, \mathrm{Ag} .1: 16.3 \%, \mathrm{Ag} .2: 12.6 \%$ of test duration; NS) whereas patch distribution was a significant factor in the other two groups (Rand: 7.4\%, Ag.1: 9.9\%, Ag.2: $10.5 \% ; P<0.10$ and Rand: $8.8 \%$, Ag.1: $12.2 \%$, Ag.2: $16.0 \% ; P<0.10)$. Further research is needed to understand such differences in the way groups exploit pastures.

\section{Effet du milieu d'élevage sur les choix alimentaires de moutons en garrigue}

\author{
S. de Rouville, J.-L. Gaubert
}

\section{Unité de zootechnie méditerranéenne, Inra, 2, place Viala, 34060 Montpellier, France}

Nous avons comparé les choix alimentaires de moutons nés et élevés en garrigue ( $G$ ) avec ceux de contemporains nés et élevés en bergerie (B) et introduits en garrigue à l'âge de 11 mois. À l'âge d'un an, cinq mâles castrés de chaque origine ont pâturé simultanément deux groupes de parcelles de $300 \mathrm{~m}^{2}$ pendant $15 \mathrm{j}$. La strate herbacée était essentiellement composée de brachypode rameux (BR) et le chêne kermès (CK) était la principale espèce ligneuse. Nous avons utilisé des transects et la méthode des points quadrats pour évaluer le taux de recouvrement (TR) de ces espèces à l'entrée et à la sortie des animaux. À l'entrée, les TR dans les parcelles pâturées par les deux lots sont identiques (BR : $50 \%[\mathrm{G}]$ versus $58 \%[\mathrm{~B}], \mathrm{CK}: 27 \%[\mathrm{G}]$ versus $25 \%[\mathrm{~B}]$ ). Lors de la $1^{\mathrm{e}}$ série de mesures, les lots ont pâturé la strate herbacée de façon semblable ( $66 \%$ [G] versus $67 \%$ [B] d'utilisation), mais les animaux élevés en garrigue ont plus consommé le chêne kermès ( $44 \%$ versus $6 \%, p<0,05)$. Lors de la $2^{\mathrm{e}}$ série de mesures, il n'y avait plus de différence d'utilisation du CK (76\% [G] versus $80 \%$ [B]). Le gain de poids des animaux au cours des périodes de mesures a été identique quelle que soit leur origine (série 1:7\% [G] versus 6\% [B], série $2: 10 \%$ [G] vs 15\% [B]). 